\title{
Global target mRNA specification and regulation by the RNA-binding protein ZFP36
}

\author{
Neelanjan Mukherjee ${ }^{1 *}$, Nicholas C Jacobs ${ }^{1}$, Markus Hafner ${ }^{3}$, Elizabeth A Kennington ${ }^{2}$, Jeffrey D Nusbaum³
} Thomas Tuschl' ${ }^{3}$ Perry J Blackshear ${ }^{2}$ and Uwe Ohler ${ }^{1 *}$

\begin{abstract}
Background: ZFP36, also known as tristetraprolin or TTP, and ELAVL1, also known as HuR, are two disease-relevant RNA-binding proteins (RBPs) that both interact with AU-rich sequences but have antagonistic roles. While ELAVL1 binding has been profiled in several studies, the precise in vivo binding specificity of ZFP36 has not been investigated on a global scale. We determined ZFP36 binding preferences using cross-linking and immunoprecipitation in human embryonic kidney cells, and examined the combinatorial regulation of AU-rich elements by ZFP36 and ELAVL1.

Results: Targets bound and negatively regulated by ZFP36 include transcripts encoding proteins necessary for immune function and cancer, and transcripts encoding other RBPs. Using partial correlation analysis, we were able to quantify the association between ZFP36 binding sites and differential target RNA abundance upon ZFP36 overexpression independent of effects from confounding features. Genes with increased mRNA half-lives in ZFP36 knockout versus wild-type mouse cells were significantly enriched for our human ZFP36 targets. We identified thousands of overlapping ZFP36 and ELAVL1 binding sites, in 1,313 genes, and found that ZFP36 degrades transcripts through specific AU-rich sequences, representing a subset of the U-rich sequences ELAVL1 interacts with to stabilize transcripts.
\end{abstract}

Conclusions: ZFP36-RNA target specificities in vivo are quantitatively similar to previously reported in vitro binding affinities. ZFP36 and ELAVL1 bind an overlapping spectrum of RNA sequences, yet with differential relative preferences that dictate combinatorial regulatory potential. Our findings and methodology delineate an approach to unravel in vivo combinatorial regulation by RNA-binding proteins.

\section{Background}

Regulation of gene expression is a complex process coordinated at many steps. Post-transcriptional regulation is controlled through RNA-binding proteins (RBPs) and non-coding RNAs (ncRNAs) interacting with RNA regulatory elements (RREs). Dynamic and combinatorial interactions of RBPs and ncRNAs with these RREs determine the functional outcome of specific steps of RNA processing, such as splicing, polyadenylation, export, stability and translation [1]. Additionally, interactions between RBPs and RREs govern messenger RNA (mRNA) stability and translational efficiency.

\footnotetext{
* Correspondence: neelanjan.mukherjee@mdc-berlin.de; uwe.ohler@mdcberlin.de

${ }^{1}$ Berlin Institute for Medical Systems Biology, Max Delbrück Center for Molecular Medicine, Robert-Rössle-Strasse 10, 13125 Berlin, Germany Full list of author information is available at the end of the article
}

AU-rich elements (AREs) are conserved cis-regulatory elements, originally discovered in the $3^{\prime}$ untranslated region (UTR) of labile mRNAs, encoding cytokines and early expressed immune response genes [2,3]. The most commonly defined AREs are the pentamer AUUUA, which is often found tandemly arranged, and the nonamer UUAUUUAUU, which is a pentamer flanked by uridylates [4-6]. AREs are generally considered instability elements, since they typically interact with RBPs that subsequently recruit mRNA degradation machinery [7,8]. Examples of ARE-binding proteins include the ELAV family, ZPF36 family, HNRNPD/AUF family, TIA1, TIAR and KSRP. Mice lacking the AU-rich region containing multiple AREs residing in the 3' UTR of the tumor necrosis factor $\alpha$ (TNF $\alpha$ ) transcript develop inflammatory disease, demonstrating their primacy in gene regulatory mechanisms [9].

\section{Biomed Central}


Tristetraprolin (ZFP36) is the founding member of the TIS11 family of RBPs. It interacts with AREs through tandem $\mathrm{CCCH}$ zinc fingers (TZFs) [10]. Mice deficient in ZFP36 also exhibit inflammatory disease phenotypes, mostly explained by deregulation of TNF $\alpha$ mRNA stability and biosynthesis [11]. The interaction of ZFP36 with AREs in the 3' UTR of targeted mRNAs, such as TNF $\alpha$, promotes mRNA degradation [12]. ZFP36 is a nucleocytoplasmic shuttling protein that is predominantly cytoplasmic at steady state [13]. The RNA-binding specificity of ZFP36 resides in the TZF domain, which interact with low nanomolar affinity with synthetic RNA substrates containing the nonamer UUAUUUAUU [14]. The NMR structure of the nonamer sequence bound to the TZF domain from human ZFP36L2 revealed that the zinc fingers symmetrically bind to UAUU half-sites, with the 5' uridylate unbound [15]. There have been many detailed in vitro investigations of ZFP36-mRNA interaction (reviewed in [16]); however, in vivo studies have only determined the mRNA pools associated with ZFP36 and have not defined individual binding sites at high resolution $[17,18]$.

Although many ARE-binding RBPs recruit mRNA degradation complexes, the ELAV/Hu family of proteins binds to AREs and promotes mRNA stability and translation [2,3,19-21]. Both in vitro and in vivo studies have determined that $\mathrm{Hu}$ proteins interact with AREs, as well as $U$ and $C U$-rich sequences [4-6,22,23]. These sequence preferences were substantiated in vivo using photoactivatable ribonucleoside cross-linking and immunoprecipitation (PAR-CLIP) to define high-resolution interaction sites for the ubiquitously expressed family member ELAVL1 [7,8,24-26]. Due to T-to-C transitions indicative of RBP-RNA interaction sites [10,27], PAR-CLIP gives a higher resolution of binding sites than earlier methods $[11,28,29]$. A commonly postulated mechanism through which ELAVL1 promotes mRNA stability is competition with ARE-binding RBPs that promote degradation. Like ZFP36, ELAVL1 is a nucleo-cytoplasmic shuttling protein, but under normal conditions is predominantly nuclear localized [12,30], consistent with numerous intronic binding sites found in PAR-CLIP experiments [13,24-26]. Simultaneous temporal and spatial competition for binding sites by ELAVL1 and ZFP36 is important when responding to stimuli, particularly immune activation, upon which ELAVL1 redistributes from the nucleus to the cytoplasm [14,31] and ZFP36 is induced and cytoplasmically localized. There is evidence for competitive binding and displacement of ZFP36 with increasing amounts of ELAVL1 and/or MAPK signaling influencing the combinatorial regulation of the TNF $\alpha$ mRNA by ELAVL1 and ZFP36 [15,32]. However, the extent to which similar competitive mechanisms exist transcriptome-wide is unknown.
Conflated direct and indirect relations are a concern, particularly when integrating multiple genomic measurements to understand complex gene regulatory mechanisms. An example of a relation relevant for post-transcriptional regulation is that longer transcripts contain more binding sites for trans-acting factors. Consequently, significant correlations between 3' UTR length and miRNA-dependent downregulation have been found in numerous datasets $[16,33]$. Overexpression of ZFP36 in the absence of stress has been demonstrated to promote stress granule assembly [18], potentially causing non-ZFP36 specific effects in transcript abundance. Partial correlation analysis quantifies the direct dependence between two variables (for example, number of binding sites or expression changes) when accounting for other variables (for example, UTR length and mRNA abundance). It has recently been applied to investigate genome evolution [34,35], as well as chromatin state and transcriptional regulation [36]. Here we apply it to account for generic transcript features when inferring functional relations between the number of binding sites and gene expression changes due to perturbing the expression of the RBP.

There are numerous examples of combinatorial binding of RBPs resulting in specific splicing decisions [37-40], but this has not been as extensively studied for mRNA stability. Given the numerous ARE-binding RBPs, the principles underlying the integration of RBP binding and concomitant regulatory outcomes have remained elusive. We therefore determined the in vivo mRNA-binding sites of ZFP36. The interaction sites and sequence preferences were compared to similarly acquired in vivo HuR interaction data, specifically contrasting AU-rich sequences with predominantly U- and CU-rich sequences. Our results indicate active competition between ZFP36 and ELAVL1 for commonly used AU-rich sequences and define the characteristics of these combinatorial regulatory events.

\section{Results and discussion}

ZFP36 preferentially binds to 3' UTRs of mRNAs encoding regulators of gene expression

We employed PAR-CLIP to identify the in vivo ZFP36 binding sites transcriptome-wide in human embryonic kidney cells (HEK293) [41]. We chose HEK293 cells and FLAG/HA-tagged ZFP36 to be as consistent as possible with previous relevant PAR-CLIP experiments with other RBPs, specifically ELAVL1. Ribonuclease-treated, immunoprecipitated and radiolabeled ribonucleoprotein (RNP) complexes resolved by sodium dodecyl sulfate polyacrylamide gel electrophoresis (SDS-PAGE) revealed a major band at approximately $50 \mathrm{kDa}$ corresponding to the FLAG/HA-tagged ZFP36 (Figure 1A). The cDNA sequence reads were processed and aligned to the human genome (see Materials and methods and Additional file 1 for detailed parameters). PARalyzer, an algorithm 


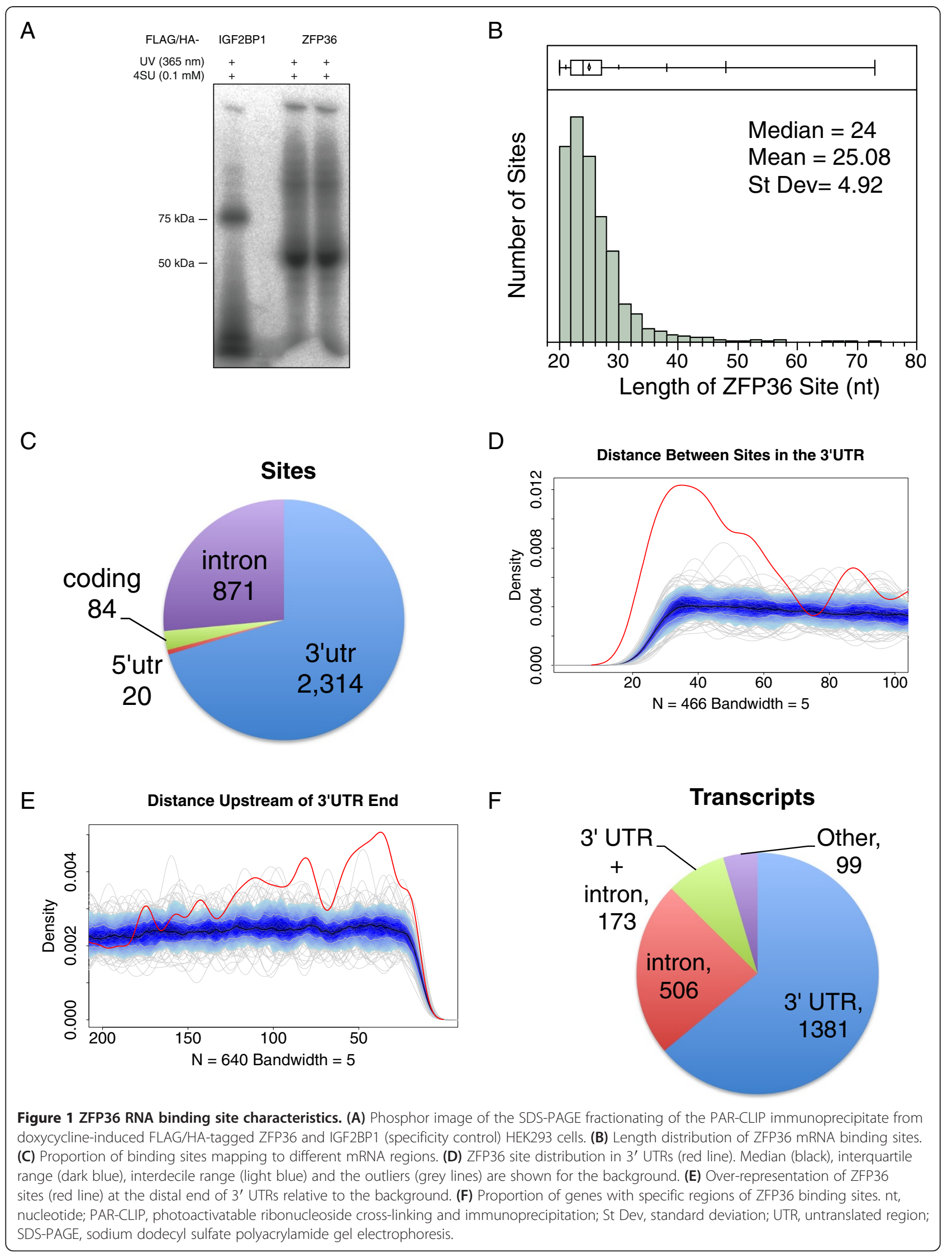


that utilizes PAR-CLIP T-to-C transitions indicative of RBP-RNA interactions, distinguished 4,626 clusters genome-wide (also referred to as binding sites, Additional file 2: Table S1) with a mean length of 25 nucleotides from 328,433 uniquely aligned T-to- $\mathrm{C}$ reconciled reads (Figure $1 \mathrm{~B}$ and Additional file 3: Figure S1A) [42]. Of the binding sites mapping to annotated and repeat-masked sequences $(3,497)$, those located in mRNA accounted for $94 \%(3,289)$.

The majority of ZFP36 mRNA binding sites (70\%, 2,314 ) mapped to the 3 ' UTR, and very few binding sites were located at the $5^{\prime}$ UTR or the protein coding region (Figure 1C). Approximately one-quarter of the mRNA binding sites were intronic (871), which is conceivable since ZFP36 is a known nucleo-cytoplasmic shuttling RBP [13]. However, the 3' UTR sites had a substantially higher $\mathrm{T}$-to- $\mathrm{C}$ conversion fraction and specificity relative to ZFP36 sites from other annotation categories including introns, 5' UTRs and protein coding regions (Additional file 3: Figure S1B). We observed closely spaced ZFP36 sites within 3' UTRs (Figure 1D), indicative of multimerization of ZFP36 and supported by results from 2-hybrid analysis and immunoreactive bands migrating at twice the expected number of kilodaltons of ZFP36 in cell extracts (PJB, unpublished data). Of the 1,148 transcripts with more than one ZFP36 site in the 3' UTR, 228 had two sites within 70 nucleotides, the range for which proximity of ZFP36 sites is above background. The statistically significant enrichment for ZFP36 sites in the last 100 nucleotides of the 3' UTR (Figure 1E) was intriguing given its ability to promote deadenylation [43]. This spatial bias was not observed for the sites of all other investigated 3' UTR binding RBPs (Additional file 3: Figure S1D) and thus not due to biases in library generation, though it may be related to AU-richness proximal to polyadenylation sites [44]. Of the 2,143 genes with ZFP36 sites, 64\% exclusively had 3' UTR sites, $23 \%$ exclusively had intronic sites and only $8 \%$ contained both 3' UTR and intronic sites (Figure 1F). Genes with 3' UTR sites were less likely to also have intronic sites in contrast to ELAVL1 [25]. The strong preference for 3' UTR binding was anticipated for an RBP predominantly involved in cytoplasmic regulation of mRNA stability and translation.

ZFP36 sites were found to be significantly enriched in transcripts encoding transcriptional and post-transcriptional regulatory proteins (Figure $2 \mathrm{~A}$ ). In addition to its own 3' UTR (Figure 2B,C), we detected ZFP36 binding to numerous sites within the 3' UTR of several other RBPs necessary for proper immune function [11,45-47], including ZFP36 family members ZFP36L1 and ZFP36L2 (five sites each; Figure 2B,D,E), ELAVL1 (two sites; Figure 2B,F), as well as $\mathrm{RC} 3 \mathrm{H} 1$ and $\mathrm{RC} 3 \mathrm{H} 2$ (two and three sites, respectively; Figure 2B). We also found that most of the
ZFP36 sites in these 3' UTRs overlapped with ELAVL1 sites (Figure 2B). The similarity of the binding domain and sequence specificity of other ZFP36 family members and the presence of both ZFP36 and ELAVL1 binding sites in these 3' UTRs warrant future investigation as a network motif that could potentially produce interesting temporal expression patterns, particularly in the context of stimuli inducing ZFP36 protein expression.

\section{In vivo ZFP36 binding specificity}

Since de novo motif finding in PARalyzer-defined ZFP36 binding sites confirmed AU-rich sequences as the most highly enriched sequence motifs (data not shown), we utilized the wealth of detailed in vitro experimental evidence for ZFP36 RNA substrate specificity (reviewed in [16]) to guide more detailed analyses. More than $84 \%$ of the mRNA sites contained known ZFP36 RREs: the classic nonamer UUAUUUAUU (8\%), the octamer UAUUUAUU (14\%) identified as the residues contacted by the TZF domain in the TIS11D structure, a single UAUU half-site (82\%), or the AUUUA pentamer (47\%). A similar fraction of intronic ZFP36 sites (804 of 871) contained one or more of the above RREs, indicating their potential for functional relevance. To assess the enrichment of each individual RRE we calculated a signalto-noise ratio (SNR). This reflects the rate of occurrence of a specific sequence in ZFP36 sites relative to the expected rate of occurrence in the background sequence, in this case within 3' UTRs (see Materials and methods for details). Each of the above RREs were found 40 to 250 times more often in the ZFP36 sites than expected by chance and were at least twice as enriched as polyUstretches of the same respective length (Figure 3A). Since the ELAVL1 library was sequenced at higher depth than the ZFP36 library, we used a size-matched subset of the ELAVL1 library using the top sites by read count $\left(E L A V L 1_{\text {best }}\right)$ to compare SNR values of various U-rich pentamer sequences in the ZFP36 to ELAVL1 PAR-CLIP data (Additional file 3: Figure S1C). The AUUUA pentamer was relatively more enriched in the ZFP36 sites, whereas UUUUU and U-stretches flanked by $\mathrm{C}$ on both or either end were relatively more enriched in ELAVL1 sites (Figure 3B). These contrasting sequence preferences are consistent with highly quantitative in vitro binding studies of both proteins, which also found that ZFP36 binds with higher affinity to AU-rich sequences relative to U-rich and CU-rich sequences, and vice versa for ELAVL1 $[48,49]$.

Previously reported in vitro ZFP36 binding studies quantified the binding of numerous RNA probes to the 73 amino acid portion of ZFP36 containing the TZF using fluorescence anisotropy measurements [48]. For each probe, we compared the experimentally determined association, $K$, and disassociation constant, $K_{d}$, with the 


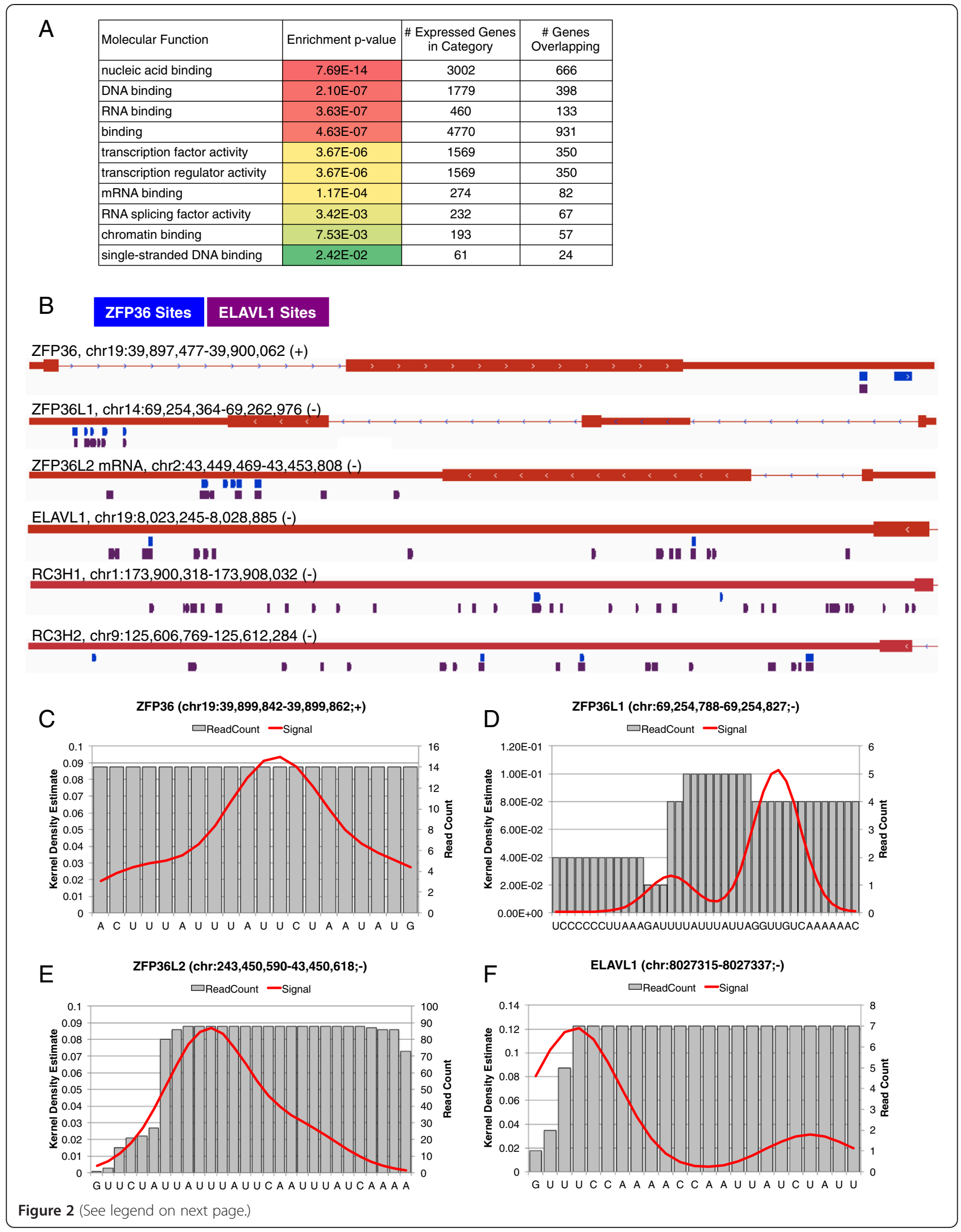


(See figure on previous page.)

Figure 2 ZFP36 binds to mRNAs encoding regulatory proteins. (A) Gene ontology enrichment analysis of genes with ZFP36 binding sites ranked and shaded from red (lowest) to green (highest) by Bonferroni corrected $P$ values $(<0.05)$ from Panther DB. (B) Distribution of ZFP36 binding sites (red) and ELAVL1 binding sites (purple) from PAR-CLIP data for immune-relevant RBPs. The genomic span is indicated for each gene and these spans vary greatly. (C-F) Visualization of individual ZFP36 binding sites with the T-to-C conversion density estimate (red line) and read counts (grey) within the ZFP36, ZFP36L1, ZFP36L2 and ELAVL1 3' UTRs. chr, chromosome; PAR-CLIP, photoactivatable ribonucleoside cross-linking and immunoprecipitation; RBP, RNA-binding protein; UTR, untranslated region.

SNR values calculated from our ZFP36 PAR-CLIP data matching experimental RNA probe and in vivo binding site sequences. We observed a very strong correlation with $K_{d}$ values (Figure $3 \mathrm{C}$ ). This strong concordance allowed us to calculate predicted $K_{d}$ values from the PAR-CLIP derived SNR ratios and thus perform in silico experiments with specific sequences. The relation between SNR and $K_{d}$ was non-linear. For example, larger differences between the SNR values for the nonamer (UUAUUUAUU) and the octamer (UUAUUUAU) translated to smaller differences in the predicted $K_{d}$ values for high affinity targets (Figure 3D). When inserting a spacer in the octamer

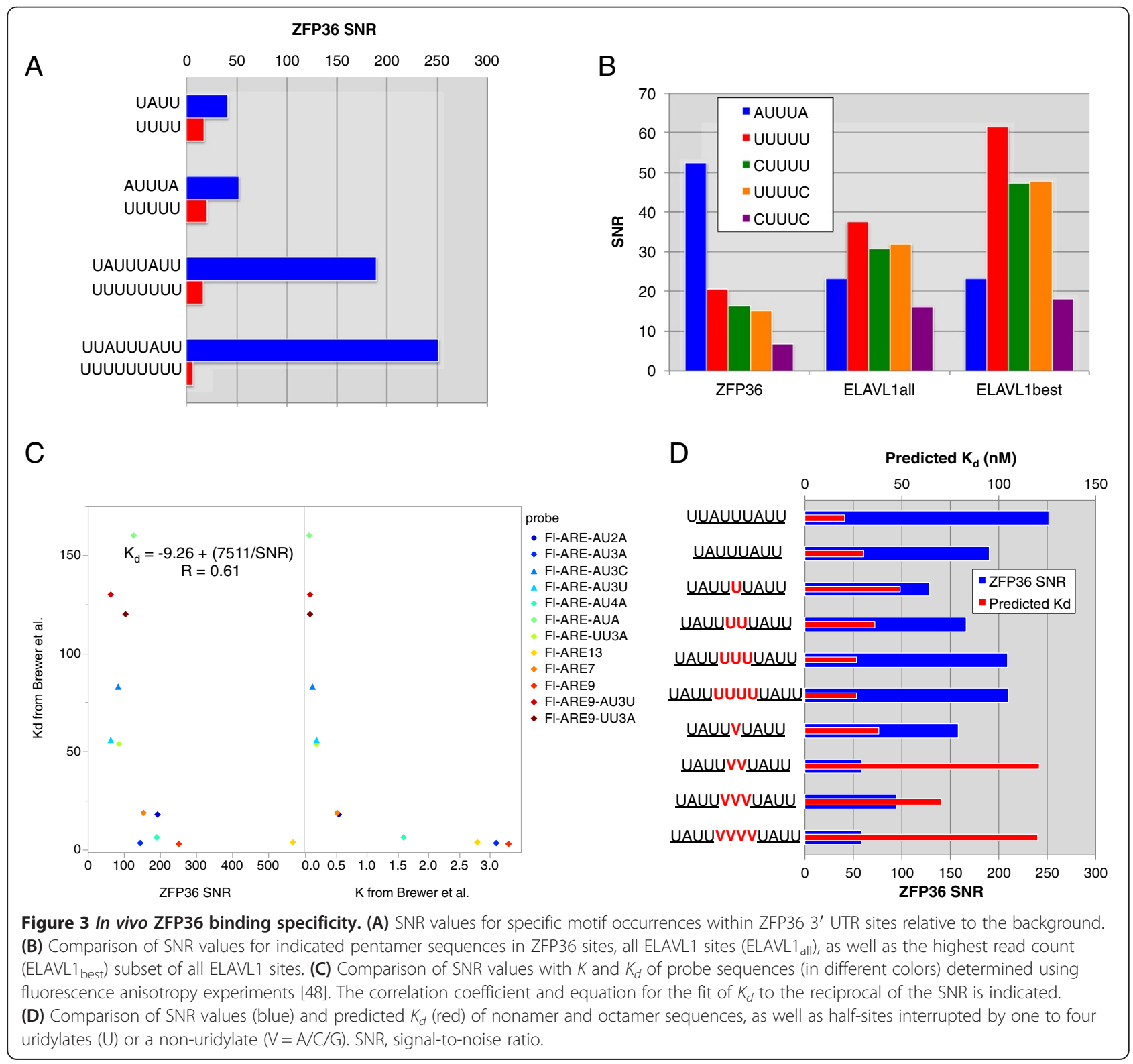


between the UUAU half-sites bound by the TZF domain, a single nucleotide disruption by any nucleotide resulted in similar increases in predicted $K_{d}$ values. However, multiple continuous disruptions with nonuridylates exhibit quite large increases in predicted $K_{d}$ values, whereas disruptions with uridylates had little to no change (Figure 3D). These results highlight the quality and complementary value of the PAR-CLIP method, as well as the detailed in vitro biochemical analysis of ZFP36 substrate specificity.

\section{Specificity of ZFP36 function}

To evaluate the regulatory function of ZFP36, we used microarray analysis to compare transcript abundance before and after doxycycline-induction of EGFP-ZFP36 fusion protein expression in HEK293 cells otherwise expressing little endogenous ZFP36 (Additional file 4: Figure S2A,B and Additional file 5: Table S2). We detected 2,784 genes with statistically significant expression differences by comparing overnight doxycycline-induced cells to mock-induced cells. Doxycycline treatment of the parental cell line did not yield any statistically significant differences defined by Bonferroni corrected $P<0.01$ (Additional file 4: Figure S2C). Only $45 \%$ of the differentially expressed genes decreased as a result of ZFP36 overexpression (downregulated genes). The large number of upregulated genes was likely due to secondary effects from prolonged ZFP36 overexpression (Additional file 4: Figure S2D). (Detailed characterization of genes with ZFP36 and ELAVL1 sites with numerous other motif contributions for significantly upregulated and downregulated genes are shown in Additional file 4: Figure S2H.) The 1,254 significantly downregulated genes decreased by approximately 3.5 fold on average. The breadth and degree of expression differences resulting from EGFP-ZFP36 overexpression were distinctly greater than the typical fine-tuning associated with most RBPs and miRNAs. This is particularly striking in comparison to ELAVL1 where only 15 genes exhibited greater than a twofold change due to ELAVL1 knockdown (Additional file 4: Figure S2G from [25]).

Downregulated genes were significantly enriched for AU-rich sequences in an unbiased analysis of the contribution of 3' UTR motif elements to expression changes (Additional file 4: Figure S2E) [50]. Thus, we examined the association between the number of counts per 3' UTR of specific AREs and EGFP-ZFP36-induced changes in mRNA abundance. The RREs described by the ZFP36L2: ARE NMR structure, the UAUUUAUU octamer and the UAUU half-site, had the strongest association with ZFP36induced changes in mRNA abundance. The UAUUUAUU octamer had a stronger association with ZFP36-induced changes in mRNA abundance than the UUAUUUAUU nonamer (Additional file 4: Figure S2F). The UAUU half-site had a stronger association with ZFP36-induced changes in mRNA abundance than the AUUUA pentamer (Additional file 4: Figure S2F).

Among differentially expressed genes, those containing ZFP36 sites were twice as often downregulated compared to those not containing ZFP36 sites (Figure 4A). Pathway enrichment analysis showed that downregulated genes with ZFP36 sites were enriched in immune and insulin signaling genes (Figure 4A). This is consistent with early studies demonstrating ZFP36-dependent immune system phenotypes as well as rapid insulin-stimulated ZFP36 induction [10,11]. Recent studies have identified that ZFP36 acts as a tumor suppressor in Myc-induced lymphomas, in which Myc directly binds to an initiator element downstream of the TATA box to suppress ZFP36 transcription. The resulting deregulation of numerous ARE-containing genes contributes to the development and maintenance of the malignant state, which can be abolished by restoring ZFP36 [51]. The top five significantly enriched pathways were remarkably accurate for the known physiological roles of ZFP36 and were not enriched for ZFP36 target genes without significant changes in expression.

To illustrate functional effects, genes are frequently stratified by a variable of interest (such as the presence or absence or number of binding sites), followed by a statistical test for significant phenotypic differences (such as expression changes) between the subsets. For instance, we observed strong associations between the number of ZFP36 sites in the 3' UTR of a gene and its downregulation due to EGFP-ZFP36 overexpression (Figure 4B), between 3' UTR length and EGFP-ZFP36-induced changes in mRNA abundance (Figure 4C), and between ZFP36 sites and 3' UTR length. These effects were corroborated by the strength of Spearman correlations between all three variables (Figure 4D, left). However, pairwise correlations are not able to quantify direct and indirect relations for more than two variables and thus we employed partial correlation analysis. Comparing the correlation and partial correlation of ZFP36 sites and 3' UTR length with EGFPZFP36-induced changes in mRNA abundance, most of the relation between ZFP36 sites and EGFP-ZFP36-induced changes in mRNA abundance was independent of 3' UTR length and vice versa (Figure 4D). Secondary effects from prolonged ZFP36 overexpression, such as spontaneous stress granule assembly [52] and changes in the expression levels of other RBPs, were potential explanations for the independent contribution of 3' UTR length to EGFPZFP36-induced changes in mRNA abundance.

We determined which transcript regions contained the most independent information for explaining EGFPZFP36-induced changes in mRNA abundance, without having to lose statistical power by separating transcripts into mutually exclusive categories, such as binding within introns only or 3' UTRs only. EGFP-ZFP36- 


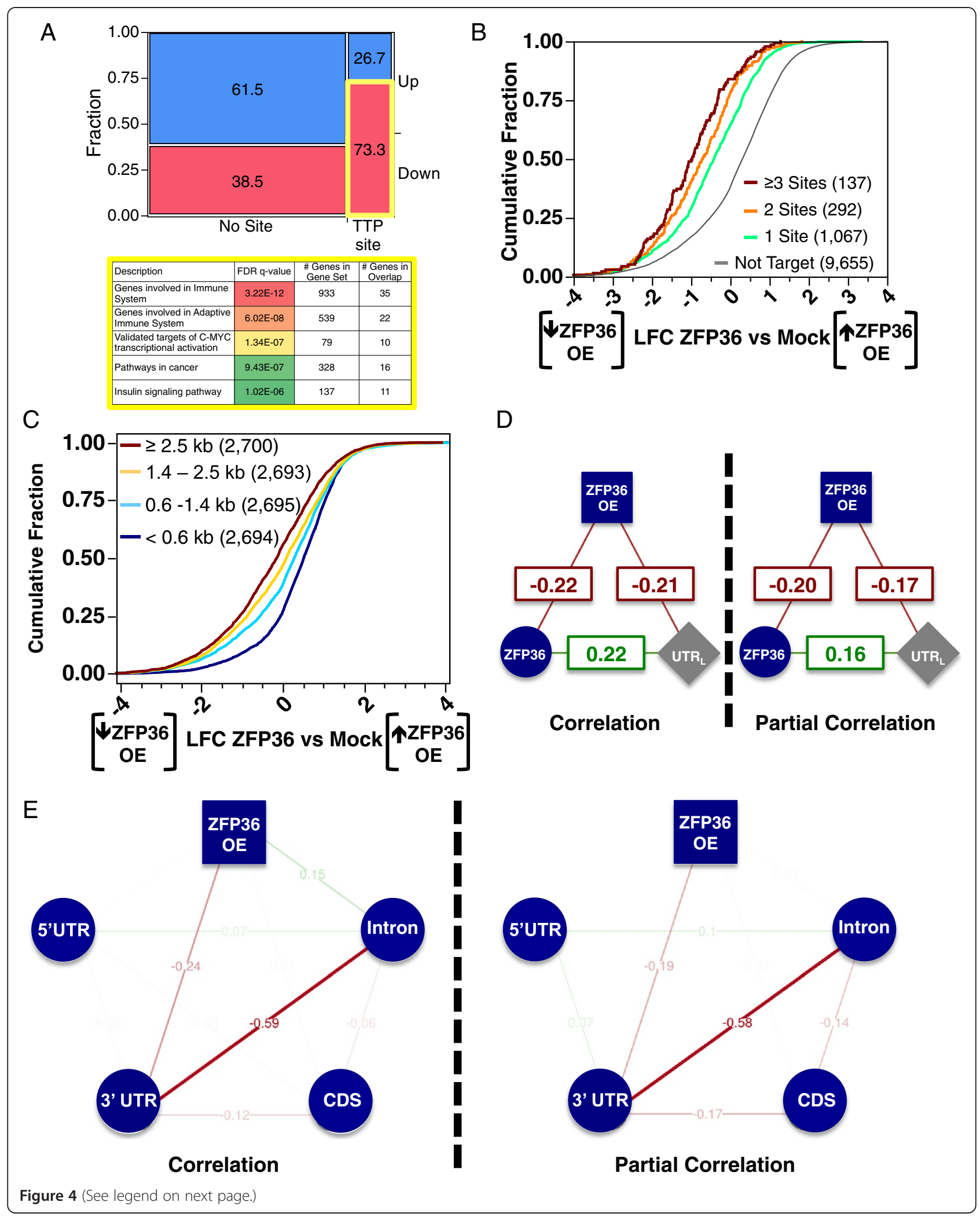


(See figure on previous page.)

Figure 4 Functional sensitivity of ZFP36 sites. (A) Mosaic plot of significantly differentially upregulated (blue) and downregulated genes (pink) with and without ZFP36 sites. Significantly enriched MSigDB canonical pathway gene sets for downregulated genes with ZFP36 sites (yellow outline). Cumulative distribution function plots of EGFP-ZFP36-induced changes in mRNA abundance for $3^{\prime}$ UTRs grouped (B) by number of ZFP36 sites and (C) by $3^{\prime}$ UTR length. For all correlation plots the color and shape of the node indicates specific data classes (circles: PAR-CLIP sites, squares: expression change upon RBP perturbation, grey diamonds: transcript features, blue: ZFP36-related feature, purple: ELAVL1-related feature). Edge line thicknesses are proportional to correlation coefficient strength and the direction of correlation is indicated by the sign and color (negative in red and positive in green). (D) Correlation (left) and partial correlation (right) analysis of number of ZFP36 sites per $3^{\prime}$ UTR (blue circles), EGFP-ZFP36-induced changes in mRNA abundance (blue squares) and 3' UTR length for all genes. (E) Correlation (left) and partial correlation (right) analysis of the number of ZFP36 sites per mRNA region and EGFP-ZFP36-induced changes in mRNA abundance for all genes with at least one ZFP36 site. kb, kilobase; PAR-CLIP, photoactivatable ribonucleoside cross-linking and immunoprecipitation; TTP, tristetraprolin; UTR, untranslated region; FDR, false discovery rate; LFC, $\log _{2}$ fold change; OE, overexpression; CDS, coding sequence.

induced changes in mRNA abundance correlated negatively with the number of ZFP36 3' UTR sites and positively with the number of intronic ZFP36 sites (Figure 4E, left). Partial correlation analysis revealed that ZFP36 sites outside of 3'UTRs, including introns, did not contribute any additional independent explanation of EGFP-ZFP36-induced changes in mRNA abundance (Figure $4 \mathrm{E}$, right). Here, the strong negative association or avoidance between the number of intronic and 3' UTR sites per gene (like the results in Figure 1F) acts as a mediator in explaining the spurious effects between intronic ZFP36 sites and EGFP-ZFP36-induced changes in mRNA abundance. This result does not rule out that ZFP36 sites from other transcript regions have other functional effects, but we only utilized 3' UTR binding sites for further analysis.

We compared our ZFP36 PAR-CLIP and overexpression data from human HEK293 cells with global mRNA stability changes after serum stimulation for ZFP36 wild-type (WT) or knockout (KO) mouse fibroblast cell lines [53]. Genes with increased mRNA half-lives in the ZFP36 KO vs WT mouse cells were significantly enriched for genes with ZFP36 PAR-CLIP sites in 3' UTR $(P=0.003)$ and genes significantly downregulated upon EGFP-ZFP36 overexpression $(P=0.001)$, but were not significantly enriched for genes significantly upregulated upon EGFP-ZFP36 overexpression $(P=0.1)$ (Additional file 6: Figure S3A). Interestingly, ELAVL1 RIP-chip targets from human Jurkat T-cell activation were significantly enriched in genes with increased mRNA half-lives in the ZFP36 KO vs WT mouse cells, even though ZFP36 RIP-chip targets from activated mouse macrophages were slightly but not significantly enriched [18]. Genes with ELAVL1 sites in the 3' UTRs were similarly enriched, but genes without ELAVL1 or ZFP36 sites in their 3' UTR did not have any enrichment. These results provide independent validation of the ZFP36 bound and regulated genes in our study using a system not reliant on ectopic overexpression of ZFP36. The enrichment of ELAVL1 gene is further support for the potential of ELAVL1 to effect expression of ZFP36 targets.

\section{Combinatorial regulation of mRNAs with AREs by ZFP36 and ELAVL1}

As could be expected given the similarity in sequence preferences, there was a substantial overlap of ZFP36 and ELAVL1 sites derived by PARalyzer from PAR-CLIP data (Figure 5A and Additional file 7: Figure S4A). Specifically, over $84 \%$ of ZFP36 sites in the 3' UTR overlapped an ELAVL1 site. Our ELAVL1 PAR-CLIP dataset corresponded well at the site level and 3' UTR level with other ELAVL1 CLIP/PAR-CLIP data in HEK93 and HeLa cell lines (Additional file 3: Figure S1E,F). Using partial correlation analysis, we compared the independent contribution of the number of ZFP36 and ELAVL1 sites to EGFP-ZFP36-induced changes in mRNA abundance, restricted to sites in the 3' UTR since these explained the vast majority of EGFP-ZFP36-induced changes in mRNA abundance (Figure 4E and Additional file 7: Figure S4B). After normalizing for differences in library size, the number of ELAVL1 sites and the number of ZFP36 sites both correlated to a similar extent with EGFP-ZFP36-induced changes in mRNA abundance (Figure 5B; cf. also Additional file 7: Figure S4C). Induction of EGFP-ZFP36 resulted in a $60 \%$ decrease in ELAVL1 mRNA levels. This decrease in ELAVL1 mRNA levels may explain some of the ability of ELAVL1 binding sites to explain ZFP36 overexpression changes. However, a previous study used a knockdown to reduce ELAVL1 protein levels to $15 \%$, and reported a much smaller degree of expression changes (Additional file 4: Figure S2G) compared to the EGFP-ZFP36-induced changes in mRNA abundance [25]. Although we cannot rule out non-linear effects from the combined increase in ZFP36 concentration and decrease in ELAVL1 concentration, the data indicate that there is substantial potential for combinatorial regulation of ARE-containing mRNAs by ZFP36 and ELAVL1.

To assess specific sequences within ELAVL1-bound sites and the strength of their contribution to the EGFPZFP36-induced changes in mRNA abundance, we performed the same analysis using ELAVL1 sites containing CUUUC, UUUUU or AUUUA. The AUUUA ELAVL1 


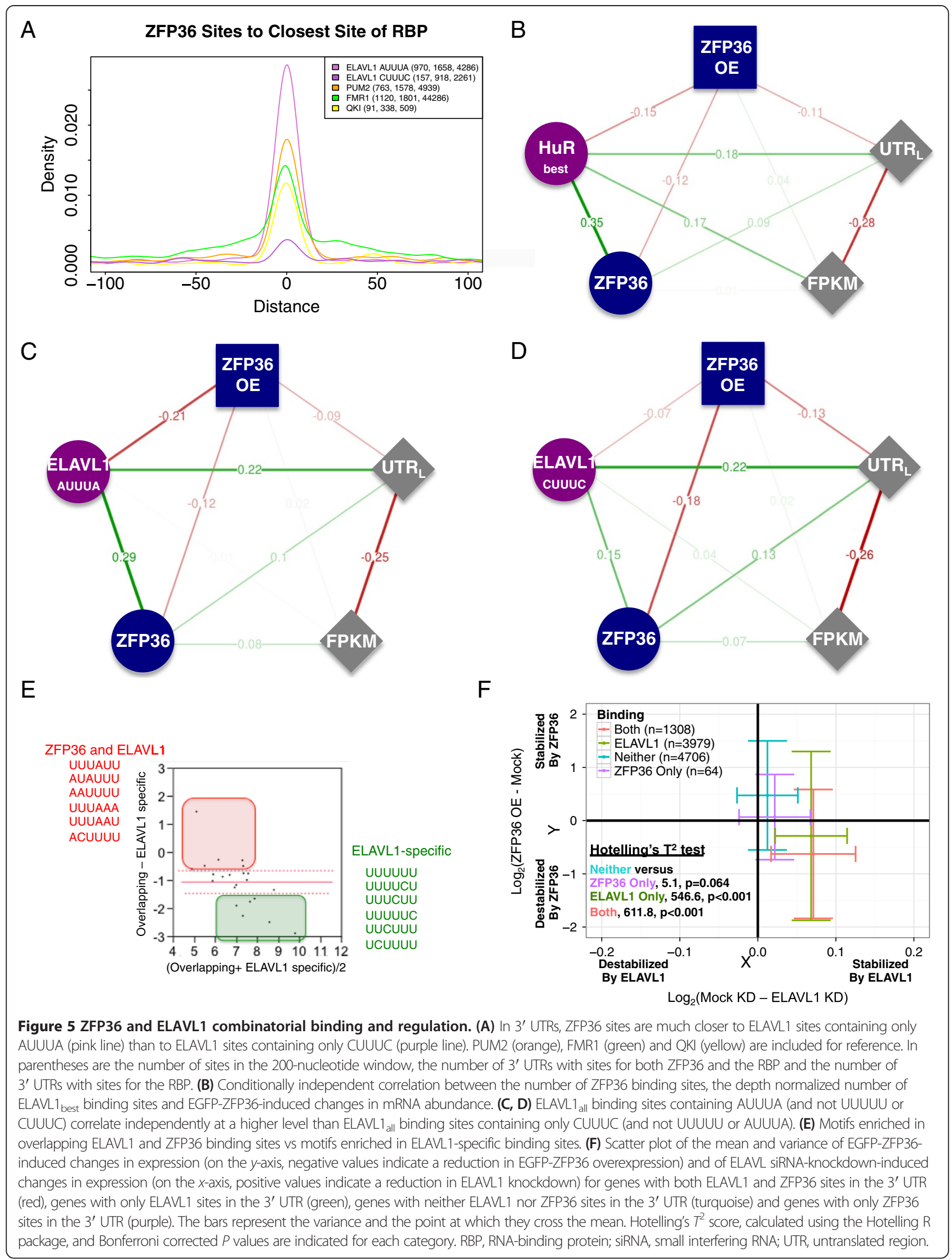


sites (-0.21) accounted for more EGFP-ZFP36-induced changes in mRNA abundance than the CUUUC ELAVL1 sites $(-0.07)$ (Figure 5C,D). This result was consistent with the observation that ELAVL1 specific sites were characterized by predominantly $\mathrm{U}$ - and $\mathrm{CU}$-rich sequences, while overlapping ELAVL1 and ZFP36 sites were characterized more by AU-rich sequences (Figure $5 \mathrm{E}$ ). The exact same analysis using an independently derived ELAVL1 HEK293 PAR-CLIP library yielded similar results (Additional file 7: Figure S4D-F), supporting the robustness of our findings. EGFP-ZFP36-induced changes in mRNA abundance were better explained by the ARE nonamer and octamer compared to $\mathrm{U}_{8}$ or $\mathrm{U}_{9}$. ELAVL1-knockdown-responsive mRNAs had the reciprocal pattern (Additional file 6: Figure S3B). Finally, transcripts containing 3' UTR sites for both ELAVL1 and ZFP36 had a greater concomitant decrease upon ELAVL1 knockdown and decrease upon ZFP36 overexpression, relative to transcripts containing 3' UTR sites for only ELAVL1 or ZFP36 or no sites (Figure 5F, shifted down and to the right). Taken together, there is substantial potential for ZFP36 and ELAVL1 to compete for the same pool of binding sites, while exerting a more targeted influence on a subset of sites with factorspecific sequence preferences.

\section{Conclusions}

Our global in vivo ZFP36-mRNA interaction experiments support previously reported biochemical in vitro ZFP36 affinity measurements, demonstrating the remarkable precision and quantitative characteristics of ZFP36 PAR-CLIP results. ZFP36 targets identified in this study were enriched for ZFP36-dependent changes in mRNA stability in mouse fibroblast cells. ZFP36 interacted with mRNAs encoding proteins important for the immune response and cancer. Many of these are other RBPs central to these two biological processes, specifically other ARE-binding RBPs such as the positive-stability regulator ELAVL1. There was extensive overlap between the interaction sites of ZFP36 and ELAVL1. Over 80\% of ZFP36 sites in 3' UTRs overlapped with ELAVL1 target sites. The preference for ZFP36 and ELAVL1 to bind and regulate mRNAs encoding RBPs suggests a complex interconnected network of post-transcriptional regulation that needs to be reconciled with respect to known ZFP36 and ELAVL1 protein expression, localization and modification dynamics, particularly in response to immune activation [54].

Partial correlation analysis accounting for confounding features demonstrated that a higher number of ZFP36 sites corresponded to stronger downregulation upon EGFPZFP36-induced changes in mRNA abundance. While the two proteins bind AREs, ZFP36 clearly shows a preferences towards AU- vs simply U-rich or CU-rich sequences relative to ELAVL1. This was corroborated at the level of mRNA stability, since ELAVL1 sites containing AUUUA were best able to explain EGFP-ZFP36-induced changes in mRNA abundance.

In summary, this study provides a resource for the precise transcriptome-wide RNA interactions of two ARE-binding RBPs with antagonistic functions. The precise sequence definition and function of AREs are an important question that will greatly benefit from global interaction data for these and other ARE-binding RBPs. Furthermore, we have integrated multiple genome-wide assays with computational analyses to advance our understanding of the sequence basis and extent of combinatorial gene regulation at the RNA level, in particular the ARE-mediated regulation by two of the most wellcharacterized RBPs.

\section{Materials and methods \\ Cell lines}

Doxycycline-inducible FLAG/HA-tagged ZFP36 HEK293 cell lines were produced utilizing the Flp- $\mathrm{In}^{\mathrm{Tm}} \mathrm{T}-\mathrm{REx}^{\mathrm{mm}} 293$ Cell Line system (Life Technologies, USA).

Flp-In $^{\text {Tw }}$ T-REx ${ }^{\text {Tw }}$ HEK293 cells were generated with a FLAG/HA-tagged human ZFP36 or an EGFP-tagged human ZFP36. Both cell lines were cultured in DMEM supplemented with $10 \%$ tetracycline-reduced fetal bovine serum and appropriate selection antibiotics. For induction of FLAG/HA-tagged or EGFP-tagged ZFP36 protein, cells were treated for $16 \mathrm{hr}$ with $1 \mu \mathrm{g} / \mathrm{ml}$ doxycycline (Sigma, USA).

\section{PAR-CLIP}

The protocol was performed as detailed in [41] for a FLAG-HA-tagged ZFP36 and sequenced using an Illumina platform. The primary dataset comprised previously derived ELAVL1 PAR-CLIP and ELAVL1 knockdown data [25]. This was integrated with ZFP36 PAR-CLIP data. An independent ELAVL1 PAR-CLIP dataset for HEK293 cells was also used to substantiate our results. Specifically, we used the union of all ELAVL1 T1 PAR-CLIP libraries. PUM2, QKI and FMR1 HEK293 PAR-CLIP libraries were investigated to compare spatial binding biases in 3' UTRs $[55,56]$. Precise analysis parameters for the PAR-CLIP libraries above are provided in Additional file 1. Binding site coordinates for ELAVL1 HEK293 CLIP and HeLa PAR-CLIP were downloaded from doRiNA [57].

Briefly, reads from the ZFP36 and ELAVL1 deepsequencing library were stripped of the adaptor sequence using Cutadapt [58]. Reads that were less than $20 \mathrm{nu}-$ cleotides in length or contained an ambiguous nucleotide were discarded. The remaining reads were aligned to the human genome (hg19), with up to one mismatch allowed and ten alignment locations, with Bowtie version 0.12 .7 [59]. Reads with T-to-C mismatches were reconciled [42] to retain only those that mapped to a single genomic location for the minimum number of mismatches. 
Annotation was performed as described in [56]. Full site- and gene-level data for the primary data compared are in Additional file 2: Table S1 and Additional file 5: Table S2, respectively.

\section{Motif affinity}

The SNR values were calculated for each probe sequence in Table one in [48] appearing at least once in 3' UTR ZFP36 binding sites. The SNR was calculated as in [42], and is defined as the number of instances per nucleotide in a given set of PARalyzer interaction sites, divided by the number of instances per nucleotide in the background set. In this case the background was the sequence of the longest annotated 3' UTR for a gene. The SNR values were plotted against the $K$ and $K_{d}$ values from [48]. The formula for the power curve fit of the SNR to $K_{d}$ was used to predict $K_{d}$ values for other sequences.

\section{Microarray samples and analysis}

EGFP-ZFP36 cells were passaged at 1:10 dilution in 15 $\mathrm{ml}$ of media in ten T75 flasks. They were allowed to grow for three days to $60 \%$ confluence. They were then treated with doxycycline (Sigma D989) to a final concentration of $1 \mu \mathrm{g} / \mathrm{ml}$. The same volume of sterile water was used as a control. The cells were incubated for $16 \mathrm{hr}$ at $37^{\circ} \mathrm{C}$, in $5 \% \mathrm{CO}_{2}$. The flasks without doxycycline were about $80 \%$ confluent. Cells with doxycycline were about $40 \%$ confluent and there were more floating cells and rounded cells. The cells were harvested by trypsinizing. Cells from five flasks per treatment were pooled. They were centrifuged at $500 \mathrm{~g}$ for $5 \mathrm{~min}$, and resuspended in growth medium to final concentrations of $1 \times 10^{7}$ cells/ml. Fluorescence-activated cell sorting (FACS) was done using a FACSAria II from Becton Dickinson (San Jose, CA). Cells were excited at $488 \mathrm{~nm}$ and sorted based on their GFP fluorescence at 525/550 nm.

The FacsDiva software version 6.1.3 was used for analysis. Doublet discrimination gates were set on forward scatter and side scatter to ensure the sorting of single cells. The sort gate was set on a histogram of GFP + cells for a fluorescence signal of greater than $10^{3} \log$ units. Control cells were sorted in the same way, except that fluorescent cells were excluded (Additional file 3: Figure S1B). The sorted cells were collected into DMEM with $10 \%$ FBS in $6 \mathrm{ml}$ polypropylene tubes at approximately $2 \times 10^{6}$ cells per tube. They were pelleted at $500 \mathrm{~g}$ for 20 $\min$ at $4^{\circ} \mathrm{C}$ and washed in PBS, then transferred into 1.5 microfuge tubes at approximately $4 \times 10^{6}$ cells per tube. These were centrifuged at $500 \mathrm{~g}$ for 20 to $30 \mathrm{~min}$ at $4^{\circ} \mathrm{C}$. The supernatant was removed, and the pellets were snap frozen in liquid nitrogen and stored at $-80^{\circ} \mathrm{C}$. This experiment was repeated on five independent days, using cells from passages 17, 20, 23, 26 and 27. RNA was isolated from approximately $3 \times 10^{6}$ to $6 \times 10^{6}$ cells using the GE Illustra RNAspin mini kit. To control for doxycycline effects, parental HEK293 cells were passaged at 1:4 or 1:5 dilution in $15 \mathrm{ml}$ medium in four T75 flasks. They grew for 2 days to approximately $60 \%$ confluence. They were then treated with doxycycline in the same way, that is, $1 \mu \mathrm{g} / \mathrm{ml}$ for $16 \mathrm{hr}$ at $37^{\circ} \mathrm{C}$ in $5 \% \mathrm{CO}_{2}$. Control cells were treated with the same volume of sterile water. The cells were harvested by trypsinizing, and processed and used for the microarray in the same way as for the GFP + cells. The same experiment was performed on five different days, and comparisons were made using five doxycycline treated vs five vehicle (water) treated samples, with each experiment done as pairs of treated and untreated cells. The samples were stored at $-80^{\circ} \mathrm{C}$. The integrity of the RNA was confirmed by running $3 \mu \mathrm{g}$ of each RNA sample on $1.2 \%$ agarose/formaldehyde gels.

Then $1.5 \mu \mathrm{g}$ of RNA from each sample was provided to the NIEHS Microarray Core Facility for analysis, using an Affymetrix Human Genome U133 Plus 2.0 gene chip (Affymetrix, Santa Clara, CA). As directed in the Affymetrix 3' IVT Express kit protocol, $100 \mathrm{ng}$ of total RNA was amplified. Then $12.5 \mu \mathrm{g}$ of amplified biotinRNA was fragmented and $10 \mu \mathrm{g}$ were hybridized to each array for $16 \mathrm{hr}$ at $45^{\circ} \mathrm{C}$ in a rotating hybridization oven using the Affymetrix Eukaryotic Target Hybridization Controls and protocol. Array slides were stained with streptavidin/phycoerythrin utilizing a double-antibody staining procedure and then washed for antibody amplification according to the GeneChip Hybridization, Wash and Stain Kit and user manual. Arrays were scanned in an Affymetrix Scanner 3000 and data were obtained using the GeneChip ${ }^{\circ}$ Command Console Software (AGCC; Version 1.1). The data were normalized using gcrma [60] and then filtered to retain only unambiguously mapping probes and minimal expression level (mean $\log$ hybridization value for replicates within a group > 4.5). Bonferroni corrected $P$ values for fold changes were calculated using Gene Pattern [61]. Gene set enrichment analysis [62] was used to compare HEK293 data to ZFP36 mouse fibroblast mRNA stability data. Human-mouse mappings were done using an appropriate microarray chip. The probes were ranked by differential mRNA half-life score, with higher scores indicating a larger slope in ZFP36 KO than ZFP36 WT cells (for details refer to [53]).

\section{Partial correlation}

Partial correlation analysis was performed using the $\mathrm{R}$ package pcor.R with Spearman correlations coefficients computed by the recursive formula [35]. Partial correlation analysis measures the degree of association between tworandom variables, independent of some set of 
other random variables. Formally, the partial correlation, $\rho X Y \mathbf{Z}$, is defined as the correlation between the residuals, $R_{X}$ and $R_{Y}$, found using linear regression of $X$ with $Z$ and $Y$ with $Z$ where $Z$ is the set of variables for which we are controlling.

$$
\begin{aligned}
& w_{X}^{*}=\arg \min _{w}\left\{\sum_{i=1}^{N}\left(x_{i}-\left\langle\mathbf{w}, \mathbf{z}_{\mathrm{i}}\right\rangle\right)^{2}\right\} \text { and } \\
& w_{Y}^{*}=\arg \min _{w}\left\{\sum_{i=1}^{N}\left(y_{i}-\left\langle\mathbf{w}, \mathbf{z}_{\mathrm{i}}\right\rangle\right)^{2}\right\}
\end{aligned}
$$

$N$ is the number of variables in the set $Z$ and $\left\langle\mathbf{w}, \mathbf{z}_{\mathbf{i}}\right\rangle$ is the scalar product of the vectors $\mathbf{w}$ and $\mathbf{z}_{\mathbf{i}}$. The residuals can then be written as:

$$
\begin{aligned}
& r_{X, i}=x_{i}-\left\langle\mathbf{w}_{\mathbf{X}}^{*}, \mathbf{z}_{\mathbf{i}}\right\rangle \\
& r_{Y, i}=y_{i}-\left\langle\mathbf{w}_{\mathbf{Y}}^{*}, \mathbf{z}_{\mathbf{i}}\right\rangle
\end{aligned}
$$

From here, the partial correlation can be obtained using the formula:

$$
\hat{\rho} X Y Z=\frac{N \sum_{i=1}^{N} r_{X, i} r_{Y, i}-\sum_{i=1}^{N} r_{X, i} \sum_{i=1}^{N} r_{Y, i}}{\sqrt{N \sum_{i=1}^{N} r_{x, i}^{2}-\left(\sum_{i-1}^{N} r_{X, i}\right)^{2}} \sqrt{N \sum_{i=1}^{N} r_{Y, i}^{2}-\left(\sum_{i-1}^{N} r_{Y, i}\right)^{2}}}
$$

It is common to use a recursive algorithm utilizing the reducibility of this $n$ th-order formula to three $(n-1)$ thorder partial correlations, represented by the formula:

$$
\rho X Y Z=\frac{\rho X Y Z \backslash\left\{Z_{0}\right\}-\rho X Y_{0} Z \backslash\left\{Z_{0}\right\} \rho Z_{0} Y Z \backslash\left\{Z_{0}\right\}}{\sqrt{1-\rho_{X Z_{0}}^{2} Z \backslash\left\{Z_{0}\right\}} \sqrt{1-\rho_{Z_{0} Y Z \backslash\left\{Z_{0}\right\}}^{2}}}
$$

$X$ is the number of ELAVL 3' UTR binding sites, $Y$ is the number of PUM2 3' UTR binding sites and $Z$ is the $\log _{2}$ change in expression due to HuR knockdown. The correlation analysis between these three variables (Figure 6, left) shows there is a positive relation between all three variables, which could lead one to believe that PUM2 binding sites could explain changes in gene expression upon HuR knockdown. However, the partial correlation or the correlation between the residuals, $R_{X}$ and $R_{Y}$ (Figure 6, right), reveals that PUM2 binding sites do not explain any additional changes in gene expression upon HuR knockdown once HuR binding sites have been accounted for. Accounting for such interdependencies is clearly important for interpreting regulatory mechanisms when integrating genomic datasets.

\section{Analysis of spatial patterns}

To visualize binding along transcripts, cluster data was taken from PARalyzer output and annotated. Only 3' UTR, 5' UTR, coding and intron annotation categories were utilized. The transcript segment associated with the annotation category of each cluster was selected using a GENCODE version 18 gene transfer format file [63]. For each gene, we used the transcript that had the longest segment matching the binding site of the cluster. The location and relevant distance along the transcript segment were then calculated. For each of the relevant annotation categories, a density plot was created utilizing each of these distances. Permutations of the observed data were used to create the background data distributions. For each cluster we created a random site along the length of its associated transcript segment repeated 100 times. The cluster plot function in the R LSD package [64], was used to plot the observed and permuted data. The LSD cluster plot function creates a graph in which the black center line is the median, and the thick gray lines separating the color gradients around the black line represent the upper and lower quartiles. The color gradient continues until the 10th and 90th percentiles.

\section{Data availability}

Both ZFP36 PAR-CLIP sequencing and overexpression microarray datasets have been deposited into GEO [GEO:GSE53185].
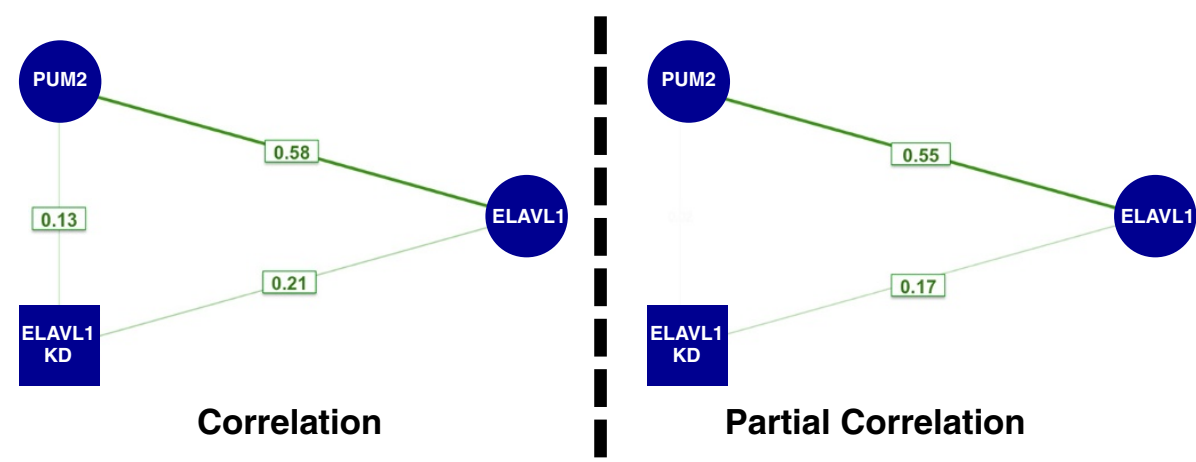

Partial Correlation

Figure 6 Correlation vs partial correlation example. Correlation (left) and partial correlation (right) analyses of number PUM2 sites per $3^{\prime}$ UTR (blue circle), ELAVL1 3' UTR sites and ELAVL1-siRNA-induced changes in mRNA abundance (blue square). siRNA, small interfering RNA; UTR, untranslated region. 


\section{Additional files}

Additional file 1: Analysis parameters. Precise analysis parameter files for ZFP36 and ELAVL1 PAR-CLIP libraries.

Additional file 2: Table S1. Comma-separated values of ZFP36 PAR-CLIP binding sites.

Additional file 3: Figure S1. ZFP36 and ELAVL1 PAR-CLIP library statistics. (A) Processing and summary statistics for reads and sites identified by ZFP36 PAR-CLIP. (B) Bubble plot comparing number of ZFP36 sites, the fraction of reads with a T-to-C conversion per cluster, and conversion specificity, which is $\log _{10}(\# T$-to- $C$ reads/(1 + \# reads with conversions not T-to-C)). (C) Number of sites in mRNA regions for primary libraries utilized (ZFP36 and ELAVL1). (D) Distribution of PAR-CLIP binding sites in length-normalized 3' UTRs for RBPs indicated by color with the counts in parentheses. (E) Distance to other ELAVL1 3' UTR sites. $(P)$ indicates the binding site coordinates were generated by our pipeline and (d) indicates the binding site coordinates were downloaded from doRiNA. PUM2 PAR-CLIP data, shown as a reference, from HEK293 cells. For each library the numbers in parentheses are the number of sites utilized in the plot, the total number of sites annotated as $3^{\prime}$ UTRs and the total number of genes containing at least one $3^{\prime}$ UTR site. (F) Venn diagram of the overlap of genes with $3^{\prime}$ UTRs with at least one binding site for the libraries indicated. Primary ELAVL1 was the dataset used throughout the current study.

Additional file 4: Figure S2. ZFP36 overexpression analysis. (A) Western blot probed with monoclonal ZFP36 antibodies demonstrating doxycycline-induced EGFP-ZFP36 expression (left two lanes) and transfection of pBluescript (BS+) or ZFP36 CDNA plasmid into HEK293 cells (right two lanes). (B) Fluorescence-activated cell sorting (FACS) analysis of EGFP-ZFP36 expression treated with vehicle (above) or doxycycline (below) see Materials and methods for details. (C) Distribution of $\log _{2}$ fold change (left) and Bonferroni corrected $P$ values (right) for ZFP36 vs mock and doxycycline vs vehicle. (D) $\log _{2}$ fold change distribution of significantly differentially expressed genes $(P<0.01)$. (E) Top enriched motifs identified by miREDUCE analysis of ZFP36 overexpression. (F) Correlation between motif occurrence in $3^{\prime}$ UTR and ZFP36 overexpression. (G) $\log _{2}$ fold change distribution for mock knockdown vs ELAVL1 knockdown. (H) Comparison between $3^{\prime}$ UTRs of transcripts significantly downregulated or upregulated upon ZFP36 overexpression by categories defined by PAR-CLIP sites with specific attributes indicated.

Additional file 5: Table S2. Comma-separated values of ZFP36 overexpression data and ZFP36 PAR-CLIP binding sites summarized at the gene level.

Additional file 6: Figure S3. Comparison of ELAVL1 and ZFP36 targets for ZFP36-deficient mRNA half-life data and known motifs. (A) Gene set enrichment analysis (GSEA) results for mouse probes ranked by score reflecting the differences in $\mathrm{mRNA}$ half-life between serum-stimulated ZFP36 KO and WT mouse fibroblasts. Name of gene set indicates data utilized, which included ZFP36 RNP immunoprecipitation-microarray (RIP-chip) targets from activated mouse macrophage RAW cells and ELAVL1 RIP-chip targets from a human Jurkat activation time course $[18,65]$. The size, normalized enrichment score (NES) and multiple hypothesis corrected significance value (FWER $P$-val) are indicated. Colored bars within each column indicate the relative value. (B) Classical ZFP36-targeted nonamers and octamers correlate more strongly with ZFP36 overexpression than strings of Us of equivalent length. The opposite relation was observed for ELAVL1 knockdown. (C) Gene ontology enrichment analysis of genes with ZFP36 binding sites ranked by Bonferroni corrected $P$ values $(<0.05)$ from the Panther DB molecular function category using the the differences in mRNA half-life scores as expression ranks.

Additional file 7: Figure S4. Further relations for ELAVL1 and ZFP36 overexpression. (A) ZFP36 sites are closer to their nearest ELAVL1 sites (blue line) than background simulations. (B) ELAVL1 binding sites in the 3' UTR are far more highly correlated with ZFP36 overexpression than those in the $5^{\prime}$ UTR, coding region or intron. (C) The number of ELAVL 1 all binding sites correlates independently with ZFP36 overexpression at a level much greater than the number of ZFP36 binding sites. Panels D, $\mathrm{E}$ and $\mathrm{F}$ utilize independently derived HEK293 ELAVL1 PAR-CLIP data from [26], which is colored a lighter purple. (D) The number of ELAVL1 binding sites found by Kishore et al. correlates independently with ZFP36 overexpression at a level much greater than the number of ZFP36 binding sites. (E, F) ELAVL1 binding sites found by Kishore et al. containing AUUUA (and not UUUUU or CUUUC) correlate independently at a higher level than ELAVL1 binding sites found by Kishroe et al. containing only CUUUC (and not UUUUU or AUUUA).

\section{Abbreviations}

ARE: AU-rich element; DMEM: Dulbecco's modified Eagle's medium; GFP: green fluorescent protein; KO: knockout; miRNA: microRNA; ncRNA: non-coding RNA; NMR: nuclear magnetic resonance; PARCLIP: photoactivatable ribonucleoside cross-linking and immunoprecipitation; PBS: phosphate-buffered saline; RBP: RNA-binding protein;

RNP: ribonucleoprotein; RRE: RNA regulatory element; siRNA: small interfering RNA; SNR: signal-to-noise ratio; TNF: tumor necrosis factor; TZF: tandem $\mathrm{CCCH}$ zinc finger; UTR: untranslated region; WT: wild type.

\section{Competing interests}

$\Pi T$ is a cofounder of and scientific advisor to Alnylam Pharmaceuticals and a scientific advisor to Regulus Therapeutics

\section{Authors' contributions}

NM, PJB and UO conceived and designed the experiments. PJB and TT designed the ZFP36 PAR-CLIP protocol. MH and JDN performed the PARCLIP. EAK and PJB designed and carried out the ZFP36 overexpression experiments. NM and NCJ performed the computational analyses. NM and UO wrote the manuscript with input from NCJ, PJB, MH and $\Pi$. All authors read and approved the final manuscript.

\section{Acknowledgements}

TT and UO acknowledge support from an award by the US National Institutes of Health (R01-GM104962). NM is supported by EU Marie Curie IIF (EU). PJB acknowledges support from the Intramural Research Program of the NIH, NIEHS. We would like to acknowledge Jack Keene for very insightful early discussions and suggestions.

\section{Author details}

${ }^{1}$ Berlin Institute for Medical Systems Biology, Max Delbrück Center for Molecular Medicine, Robert-Rössle-Strasse 10, 13125 Berlin, Germany. ${ }^{2}$ Laboratory of Signal Transduction, National Institute of Environmental Health Sciences, Research Triangle Park, NC 27709 USA. ${ }^{3}$ Howard Hughes Medical Institute and Laboratory for RNA Molecular Biology, The Rockefeller University, New York, NY 10065 USA.

Received: 6 August 2013 Accepted: 8 January 2013

Published: 8 January 2014

\section{References}

1. Keene JD: RNA regulons: coordination of post-transcriptional events. Nat Rev Genet 2007, 8:533-543.

2. Caput D, Beutler B, Hartog K, Thayer R, Brown-Shimer S, Cerami A: Identification of a common nucleotide sequence in the 3 '-untranslated region of $m R N A$ molecules specifying inflammatory mediators. Proc Natl Acad Sci USA 1986, 83:1670-1674.

3. Shaw $G$, Kamen R: A conserved AU sequence from the 3' untranslated region of GM-CSF mRNA mediates selective mRNA degradation. Cell 1986, 46:659-667.

4. Xu N, Chen CY, Shyu AB: Modulation of the fate of cytoplasmic mRNA by $\mathrm{AU}$-rich elements: key sequence features controlling mRNA deadenylation and decay. Mol Cell Biol 1997, 17:4611-4621.

5. Zubiaga AM, Belasco JG, Greenberg ME: The nonamer UUAUUUAUU is the key AU-rich sequence motif that mediates mRNA degradation. Mol Cell Biol 1995, 15:2219-2230.

6. Bakheet T, Williams BRG, Khabar KSA: ARED 3.0: the large and diverse AU-rich transcriptome. Nucleic Acids Res 2006, 34:D111-D114. 
7. Shyu AB, Belasco JG, Greenberg ME: Two distinct destabilizing elements in the $c$-fos message trigger deadenylation as a first step in rapid mRNA decay. Genes Dev 1991, 5:221-231.

8. Chen CY, Gherzi R, Ong SE, Chan EL, Raijmakers R, Pruijn GJ, Stoecklin G, Moroni C, Mann M, Karin M: AU binding proteins recruit the exosome to degrade ARE-containing mRNAs. Cell 2001, 107:451-464

9. Kontoyiannis D, Pasparakis M, Pizarro TT, Cominelli F, Kollias G: Impaired on/ off regulation of TNF biosynthesis in mice lacking TNF AU-rich elements. Cell 1999, 10:387-398.

10. Lai WS, Stumpo DJ, Blackshear PJ: Rapid insulin-stimulated accumulation of an mRNA encoding a proline-rich protein. J Biol Chem 1990, 265:16556-16563.

11. Taylor GA, Carballo E, Lee DM, Lai WS, Thompson MJ, Patel DD, Schenkman DI, Gilkeson GS, Broxmeyer HE, Haynes BF, Blackshear PJ: A pathogenetic role for TNFa in the syndrome of cachexia, arthritis, and autoimmunity resulting from tristetraprolin (TTP) deficiency. Cell 1996, 4:445-454.

12. Carballo E: Feedback inhibition of macrophage tumor necrosis factor-a production by tristetraprolin. Science 1998, 281:1001-1005

13. Phillips RS: Members of the tristetraprolin family of tandem $\mathrm{CCCH}$ zinc finger proteins exhibit CRM1-dependent nucleocytoplasmic shuttling. J Biol Chem 2002, 277:11606-11613.

14. Blackshear PJ, Lai WS, Kennington EA, Brewer G, Wilson GM, Guan X, Zhou $P$ : Characteristics of the interaction of a synthetic human tristetraprolin tandem zinc finger peptide with AU-rich element-containing RNA substrates. J Biol Chem 2003, 278:19947-19955.

15. Hudson BP, Martinez-Yamout MA, Dyson HJ, Wright PE: Recognition of the mRNA AU-rich element by the zinc finger domain of TIS11d. Nat Struct Mol Biol 2004, 11:257-264

16. Brooks SA, Blackshear PJ: Tristetraprolin (TTP): interactions with mRNA and proteins, and current thoughts on mechanisms of action. Biochim Biophys Acta (BBA) - Gene Regul Mech 2013, 1829:666-679.

17. Emmons J, Townley-Tilson WHD, Deleault KM, Skinner SJ, Gross RH, Whitfield ML, Brooks SA: Identification of TTP mRNA targets in human dendritic cells reveals TTP as a critical regulator of dendritic cell maturation. RNA 2008, 14:888-902.

18. Stoecklin G, Tenenbaum SA, Mayo T, Chittur SV, George AD, Baroni TE, Blackshear PJ, Anderson P: Genome-wide analysis identifies interleukin-10 mRNA as target of tristetraprolin. J Biol Chem 2008, 283:11689-11699.

19. Jain RG, Andrews LG, McGowan KM, Pekala PH, Keene JD: Ectopic expression of Hel-N1, an RNA-binding protein, increases glucose transporter (GLUT1) expression in 3 T3-L1 adipocytes. Mol Cell Biol 1997, 17:954-962.

20. Fan XC, Myer VE, Steitz JA: AU-rich elements target small nuclear RNAs as well as mRNAs for rapid degradation. Genes Dev 1997, 11:2557-2568.

21. Peng SS, Chen $C Y, X u N$, Shyu AB: RNA stabilization by the AU-rich element binding protein, HuR, an ELAV protein. EMBO J 1998, 17:3461-3470

22. Levine TD, Gao F, King PH, Andrews LG, Keene JD: Hel-N1: an autoimmune RNA-binding protein with specificity for 3 ' uridylate-rich untranslated regions of growth factor mRNAs. Mol Cell Biol 1993, 13:3494-3504.

23. Tenenbaum SA, Carson CC, Lager PJ, Keene JD: Identifying mRNA subsets in messenger ribonucleoprotein complexes by using cDNA arrays. Proc Natl Acad Sci USA 2000, 97:14085-14090.

24. Lebedeva S, Jens M, Theil K, Schwanhäusser B, Selbach M, Landthaler M, Rajewsky N: Transcriptome-wide analysis of regulatory interactions of the RNA-binding protein HuR. Mol Cell 2011, 43:340-352.

25. Mukherjee N, Corcoran DL, Nusbaum JD, Reid DW, Georgiev S, Hafner M Ascano M, Tuschl T, Ohler U, Keene JD: Integrative regulatory mapping indicates that the RNA-binding protein HuR couples pre-mRNA processing and mRNA stability. Mol Cell 2011, 43:327-329.

26. Kishore S, Jaskiewicz L, Burger L, Hausser J, Khorshid M, Zavolan M: A quantitative analysis of CLIP methods for identifying binding sites of RNA-binding proteins. Nat Meth 2011, 8:559-564.

27. Hafner M, Max KEA, Bandaru P, Morozov P, Gerstberger S, Brown M, Molina $H$, Tuschl T: Identification of mRNAs bound and regulated by human LIN28 proteins and molecular requirements for RNA recognition. RNA 2013, 19:613-626.

28. Natarajan A, Yardımcı GGR, Sheffield NC, Crawford GE, Ohler U: Predicting cell-type-specific gene expression from regions of open chromatin. Genome Res 2012, 22:1711-1722.

29. Ule J, Jensen KB, Ruggiu M, Mele A, Ule A, Darnell RB: CLIP identifies Nova-regulated RNA networks in the brain. Science 2003, 302:1212-1215.
30. Fan XC, Steitz JA: HNS, a nuclear-cytoplasmic shuttling sequence in HuR. Proc Natl Acad Sci USA 1998, 95:15293-15298.

31. Atasoy U, Watson J, Patel D, Keene JD: ELAV protein HuA (HuR) can redistribute between nucleus and cytoplasm and is upregulated during serum stimulation and T cell activation. J Cell Sci 1998, 111:3145-3156.

32. Tiedje C, Ronkina N, Tehrani M, Dhamija S, Laass K, Holtmann H, Kotlyarov A, Gaestel M: The p38/MK2-driven exchange between tristetraprolin and HuR regulates AU-rich element-dependent translation. PLOS Genet 2012, 8:e1002977.

33. Arvey A, Larsson E, Sander C, Leslie CS, Marks DS: Target mRNA abundance dilutes microRNA and siRNA activity. Mol Syst Biol 2010, 6: [http://www. nature.com/msb/journal/v6/n1/full/msb201024.html]

34. Freudenberg J, Wang M, Yang Y, Li W: Partial correlation analysis indicates causal relationships between GC-content, exon density and recombination rate in the human genome. BMC Bioinformatics 2009, 10:S66.

35. Kim SH: Correlated asymmetry of sequence and functional divergence between duplicate proteins of saccharomyces cerevisiae. Mol Biol Evol 2006, 23:1068-1075.

36. Gat-Viks I, Vingron M: Evidence for gene-specific rather than transcription rate-dependent histone $\mathrm{H} 3$ exchange in yeast coding regions. PloS Comp Biol 2009, 5:e1000282.

37. Barash Y, Calarco JA, Gao W, Pan Q, Wang X, Shai O, Blencowe BJ, Frey BJ: Deciphering the splicing code. Nature 2010, 465:53-59.

38. Pandit S, Zhou Y, Shiue L, Coutinho-Mansfield G, Li H, Qiu J, Huang J, Yeo GW, Ares M, Fu X-D: Genome-wide analysis reveals SR protein cooperation and competition in regulated splicing. Mol Cell 2013, 50:223-235.

39. Zarnack K, König J, Tajnik M, Martincorena I, Eustermann S, Stévant I, Reyes A, Anders S, Luscombe NM, Ule J: Direct competition between hnRNP C and U2AF65 protects the transcriptome from the exonization of Alu elements. Cell 2013, 152:453-466.

40. Zhang C, Frias MA, Mele A, Ruggiu M, Eom T, Marney CB, Wang H, Licatalosi DD, Fak JJ, Darnell RB: Integrative modeling defines the Nova splicingregulatory network and its combinatorial controls. Science 2010, 329:439-443.

41. Hafner M, Landthaler M, Burger L, Khorshid M, Hausser J, Berninger $P$, Rothballer A, Ascano M, Jungkamp A-C, Munschauer M, Ulrich A, Wardle GS, Dewell S, Zavolan M, Tuschl T: Transcriptome-wide identification of RNA-binding protein and microRNA target sites by PAR-CLIP. Cell 2010, 141:129-141.

42. Corcoran DL, Georgiev S, Mukherjee N, Gottwein E, Skalsky RL, Keene JD, Ohler U: PARalyzer: definition of RNA binding sites from PAR-CLIP short-read sequence data. Genome Biol 2011, 12:R79.

43. Fabian MR, Frank F, Rouya C, Siddiqui N, Lai WS, Karetnikov A, Blackshear PJ, Nagar B, Sonenberg N: Structural basis for the recruitment of the human CCR4-NOT deadenylase complex by tristetraprolin. Nat Struct Mol Biol 2013, 20:735-739.

44. Pesole G, Liuni S, Grillo G, Saccone C: Structural and compositional features of untranslated regions of eukaryotic mRNAs. Gene 1997 205:95-102.

45. Leppek K, Schott J, Reitter S, Poetz F, Hammond MC, Stoecklin G: Roquin promotes constitutive mRNA decay via a conserved class of stem-loop recognition motifs. Cell 2013, 153:869-881.

46. Hodson DJ, Janas ML, Galloway A, Bell SE, Andrews S, Li CM, Pannell R, Siebel CW, MacDonald HR, De Keersmaecker K, Ferrando AA, Grutz G, Turner M: Deletion of the RNA-binding proteins ZFP36L1 and ZFP36L2 leads to perturbed thymic development and T lymphoblastic leukemia. Nat Immunol 2010, 11:717-724

47. Papadaki O, Milatos S, Grammenoudi S, Mukherjee N, Keene JD, Kontoyiannis DL: Control of thymic T cell maturation, deletion and egress by the RNA-binding protein HuR. J Immunol 2009, 182:6779-6788.

48. Brewer BY: RNA sequence elements required for high affinity binding by the zinc finger domain of tristetraprolin: conformational changes coupled to the bipartite nature of AU-rich mRNA-destabilizing motifs. J Biol Chem 2004, 279:27870-27877.

49. Meisner N-C, Hackermüller J, Uhl V, Aszódi A, Jaritz M, Auer M: mRNA openers and closers: modulating AU-rich element-controlled mRNA stability by a molecular switch in mRNA secondary structure. ChemBioChem 2004, 5:1432-1447.

50. Sood P: Cell-type-specific signatures of microRNAs on target mRNA expression. Proc Natl Acad Sci USA 2006, 103:2746-2751. 
51. Rounbehler RJ, Fallahi M, Yang C, Steeves MA, Li W, Doherty JR, Schaub FX, Sanduja S, Dixon DA, Blackshear PJ, Cleveland JL: Tristetraprolin impairs Myc-induced lymphoma and abolishes the malignant state. Cell 2012, 150:563-574.

52. Stoecklin G, Stubbs T, Kedersha N, Wax S, Rigby WF, Blackwell TK, Anderson P: MK2-induced tristetraprolin:14-3-3 complexes prevent stress granule association and ARE-mRNA decay. Nat Struct Mol Biol 2004, 23:1313-1324.

53. Lai WS, Parker JS, Grissom SF, Stumpo DJ, Blackshear PJ: Novel mRNA targets for tristetraprolin (TTP) identified by global analysis of stabilized transcripts in TTP-deficient fibroblasts. Mol Cell Biol 2006, 26:9196-9208.

54. Kratochvill F, Machacek C, Vogl C, Ebner F, Sedlyarov V, Gruber AR, Hartweger H, Vielnascher R, Karaghiosoff M, Rülicke T, Müller M, Hofacker I, Lang R, Kovarik P: Tristetraprolin-driven regulatory circuit controls quality and timing of mRNA decay in inflammation. Mol Syst Bio/ 2011, 7: [http://www.nature.com/msb/journal/v7/n1/synopsis/msb201193.html]

55. Hafner M, Lianoglou S, Tuschl T, Betel D: Genome-wide identification of miRNA targets by PAR-CLIP. Methods 2012, 58:94-105.

56. Ascano M, Mukherjee N, Bandaru P, Miller JB, Nusbaum JD, Corcoran DL, Langlois C, Munschauer M, Dewell S, Hafner M, Williams Z, Ohler U, Tuschl T: FMRP targets distinct mRNA sequence elements to regulate protein expression. Nature 2012, 492:382-386.

57. Anders G, Mackowiak SD, Jens M, Maaskola J, Kuntzagk A, Rajewsky N, Landthaler M, Dieterich C: doRiNA: a database of RNA interactions in post-transcriptional regulation. Nucleic Acids Res 2012, 40:D180-D186

58. Martin M: Cutadapt removes adapter sequences from high-throughput sequencing reads. EMBnet I 2011, 17:10-12.

59. Langmead B, Trapnell C, Pop M, Salzberg SL: Ultrafast and memory-efficient alignment of short DNA sequences to the human genome. Genome Biol 2009, 10:R25.

60. Wu Z, Irizarry RA, Gentleman R, Murillo FM, Spencer F: A model based background adjustment for oligonucleotide expression arrays. J Am Stat Assoc 2004, 99:909.

61. Gould J, Getz G, Monti S, Reich M, Mesirov JP: Comparative gene marker selection suite. Bioinformatics 2006, 22:1924-1925.

62. Subramanian A, Tamayo P, Mootha VK, Mukherjee S, Ebert BL, Gillette MA, Paulovich A, Pomeroy SL, Golub TR, Lander ES, Mesirov JP: Gene set enrichment analysis: a knowledge-based approach for interpreting genome-wide expression profiles. Proc Natl Acad Sci USA 2005, 102:15545-15550

63. GENCODE project. [http://www.gencodegenes.org/releases/18.html]

64. LSD: Lots of Superior Depictions. [http://CRAN.R-project.org/package=LSD]

65. Mukherjee N, Lager PJ, Friedersdorf MB, Thompson MA, Keene JD: Coordinated posttranscriptional mRNA population dynamics during T-cell activation. Mol Syst Biol 2009, 5:288.

doi:10.1186/gb-2014-15-1-r12

Cite this article as: Mukherjee et al:: Global target mRNA specification and regulation by the RNA-binding protein ZFP36. Genome Biology 2014 15:R12.

\section{Submit your next manuscript to BioMed Central and take full advantage of:}

- Convenient online submission

- Thorough peer review

- No space constraints or color figure charges

- Immediate publication on acceptance

- Inclusion in PubMed, CAS, Scopus and Google Scholar

- Research which is freely available for redistribution 